\title{
Michel Arrivé
}

(1936-2017)

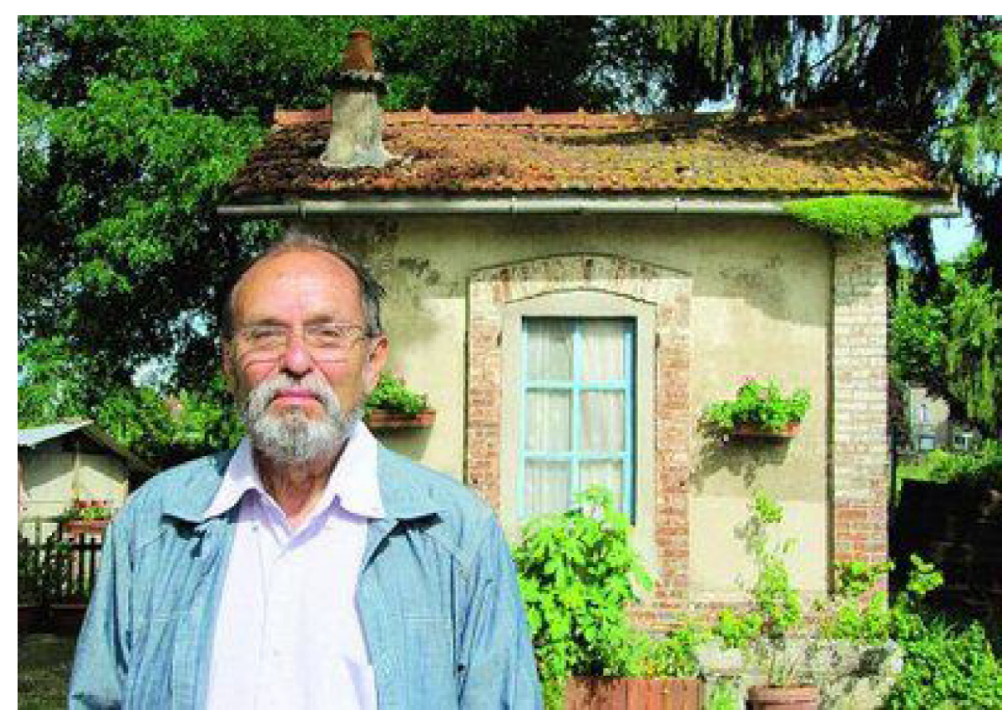

«Le mot, c'est la mort sans en avoir l'air»

M. Arrivé, Les remembrances du vieillard idiot, Flammarion 1977, épigraphe.

«Le mot, c'est la mort sans en avoir l'R»

Adolphe Ripotois, cité par M. Arrivé, Langage et psychanalyse, linguistique et inconscient, Freud, Saussure, Pichon, Lacan, PUF, 1994, p. 119.

Michel Arrivé, Professeur émérite de l'université Paris Nanterre, est décédé brutalement le 3 avril 2017. Avec retard (du fait de contraintes éditoriales), l'UMR 7597 et la revue Histoire Épistémologie Langage présentent à sa famille ses sincères condoléances.

Les hommages et nécrologies qui ont suivi la triste nouvelle n'ont pas manqué de mettre en évidence la diversité des centres d'intérêt et d'activité d'un grammairien «atypique ». 
Mais existe-t-il un idéal-type du grammairien, hier comme aujourd'hui?

Grammairien donc, (plus jeune agrégé de sa génération - il n'a été égalé dans cette performance que par le regretté Jacques Anis dont il dirigera la thèse plusieurs décennies plus tard), M. Arrivé a participé à l'essor de ce qu'on a pu appeler l'École Sémiotique de Paris dans la lignée de A.J. Greimas. Il a largement participé dans les années 60/70, au sein d'institutions dont la mémoire s'est aujourd'hui perdue (la Société d'Étude de Langue Française, par exemple) ou, ensuite et pour de longues années, à la Présidence de la très officielle $7^{\mathrm{e}}$ section du Comité National des Universités, à l'installation et au développement de filières renouvelées d'enseignement et de recherche en sciences du langage. Avec d'autres sans doute, à la fois avec discrétion et autorité, il a œuvré au destin professionnel de nombreux linguistes dans tous les champs des sciences du langage. Ils lui sont tous, à des degrés divers, redevables.

Souvenons-nous d'un temps pas si éloigné (les années 50/60 du siècle précédent): les sciences du langage (ce que nous appelons ainsi aujourd'hui) n'existaient que par truchement. Ce qu'on a appelé la linguistique - en particulier la linguistique générale - ne se distinguait alors pas de la philologie dans des départements de Lettres ou de langue (littérature et civilisation). Longue histoire très française (mais pas unique non plus en Europe) qui voit la première chaire universitaire de « linguistique générale » ouverte à La Sorbonne en 1955 seulement pour A. Martinet, au lieu même de précipitation pourtant de la prestigieuse École linguistique de Paris (M. Bréal, G. Paris, A. Meillet, F. de Saussure, M. Cohen, J. Vendryes, É. Benveniste...) avec l'EPHE comme machine de guerre, et le Collège de France comme avant-garde... à la charnière des $\mathrm{XIX}^{\mathrm{e}}$ et $\mathrm{XX}^{\mathrm{e}}$ siècles.

Mais les héritiers indociles ne sont pas les moins fidèles et M. Arrivé, Assistant du très conservateur Professeur «en Sorbonne»F. Deloffre après un début de carrière dans un collège de la banlieue parisienne, rejoindra donc la prestigieuse Maison pour trouver après mai 1968, dans la sulfureuse université de Nanterre, le lieu naturel propice à sa créativité.

Ses activités se répartissent en de nombreux pôles indissociablement éditoriaux et pédagogiques (ici sans exhaustivité) :

- les travaux sur Alfred Jarry: Les langages de Jarry, essai de sémiotique littéraire (1972) qui inaugure sa participation active à l'école de sémiotique, et Lire Jarry (1976);

- les travaux de grammaire et de linguistique française: rédaction de la Grammaire Larousse du français contemporain (1964) avec J.-C. Chevalier, C.-B. Benveniste, J. Peytard, La grammaire d'aujourd'hui, guide alphabétique de linguistique française (1986) avec F. Gadet et M. Galmiche, Réformer l'orthographe (1993), Verbes sages et verbes fous (2005), Formes sémiotiques (2008); 
- les relations entre langage et inconscient notamment: Langage et psychanalyse (2006), Le linguiste et l'inconscient (2008), Régimes sémiotiques de la temporalité (2006). Dans ce domaine, on peut dire que M. Arrivé aura été le premier et sans doute le seul à affronter de manière à la fois modeste et claire un champ de la modernité souvent encombré de certitudes approximatives et tonitruantes. Avec une attention "vétilleuse» - son adjectif préféré - il aura largement contribué de manière rigoureuse à démêler l'imbroglio «structuraliste» et à défaire bien des légendes autour de la «linguistiquerie» lacanienne. Sa connaissance profonde des textes de Freud, conjointe à celle de ceux de Saussure, ont une puissance démystificatrice salutaire et une force pionnière dans l'archéologie des sciences humaines du $\mathrm{xx}^{\mathrm{e}}$ siècle.

Mais c'est sans doute dans le domaine saussurien ou «saussurologique» que le talent de M. Arrivé à trouvé ces dernières années son champ d'exercice le plus dynamique. Avec sa trilogie À la recherche de Ferdinand de Saussure (2007), Du côté de chez Saussure (ouvrage collectif, Lambert-Lucas 2008), Saussure retrouvé (2016), Michel Arrivé a pu croiser les différents secteurs de ses activités, qu'il s'agisse de grammaire (Saussure et la syntaxe, par exemple), de littérature (De la lettre à la littérature. Jarry, Saussure, Roussel et quelques autres, 2016), de sémiotique, des développements sur les destins parallèles de la notion d'inconscient en psychanalyse et en linguistique. Parfait connaisseur du «nouveau Saussure»-celui des inédits et de la transmission indirecte des «cours »-, M. Arrivé ne croyait guère en un néo-saussurisme. Il pensait plutôt que la découverte des nouveaux textes permettait au contraire d'en finir avec la prolifération illusoire des figures de Saussure. Du Mémoire aux Anagrammes, aux Légendes germaniques, aux traités de phonétique ou de versification française, aux notes sur l'audition colorée etc., il y a une unité profonde non évidente, mais d'autant plus stimulante qu'elle est cachée. C'est à cette unité que s'attachait l'enquête «vétilleuse» de M. Arrivé sous l'égide de ses titres proustiens.

C'est aussi que l'histoire de la linguistique a accompagné toute sa carrière et le développement de sa pensée. L'ouvrage de 1970 La grammaire: lectures (avec J.-C. Chevalier), inaugurait un cheminement historique de plus large empan que la saussurologie tardive. M. Arrivé connaissait la tradition grammaticale française, fréquentait les héritiers de la grammaire générale, cultivait le gout pour des grammairiens mal connus mais inventifs. Il a fait beaucoup pour la redécouverte du «monstre dicéphale» (Arrivé dixit) si singulier que représentent Damourette et Pichon, linguistes et métalinguistes propres à stimuler l'imaginaire rationnel du pataphysicien. 
M. Arrivé a été l'un des premiers abonnés de la revue Histoire Épistémologie Langage, il a participé en tant que membre particulièrement actif aux activités de la Société d'histoire et épistémologie des sciences du langage, il a toujours soutenu dans les institutions l'idée que l'histoire de la linguistique était de la linguistique (de la linguistique sui-réflexive), et la linguistique une activité spécialisée et technique qui ne pouvait ignorer sa propre histoire sans risquer de se perdre.

Nous perdons avec M. Arrivé un collègue, un ami, un Professeur, un soutien.

Nous perdons également un compagnon de rire de très bon aloi :

«Car le rire pour Saussure est la condition préalable du sérieux de la linguistique» ( «Ce qui fait rire le linguiste, ou du métalangage chez Saussure», Saussure retrouvé, p. 21)

Ripotois, avatar pataphysique de M. Arrivé, personnage récurrent de plusieurs notes des ouvrages les plus sérieux de Michel Arrivé tout au long de sa carrière, ne nous démentirait certainement pas.

Christian Puech 